\title{
Adaptive Synchronization via State Predictor on General Complex Dynamic Networks
}

\author{
Lijun Wang, ${ }^{1}$ Pingnan Ruan, ${ }^{1}$ Shuang Li, ${ }^{2}$ and Xiao Han ${ }^{2}$ \\ ${ }^{1}$ School of Economics and Management, Beijing University of Technology, Beijing 100022, China \\ ${ }^{2}$ College of Science, North China University of Technology, Beijing 100144, China \\ Correspondence should be addressed to Shuang Li; 374915249@qq.com
}

Received 25 June 2014; Accepted 14 August 2014

Academic Editor: Michael Chen

Copyright (c) 2015 Lijun Wang et al. This is an open access article distributed under the Creative Commons Attribution License, which permits unrestricted use, distribution, and reproduction in any medium, provided the original work is properly cited.

\begin{abstract}
This paper considers the adaptive synchronization of general complex dynamic networks via state predictor based on the fixed topology for nonlinear dynamical systems. Using Lyapunov stability properties, it is proved that the complex dynamical networks with state predictor are asymptotically stable. Moreover, it is also shown that the rate of convergence of complex dynamical networks with state predictor is faster than the complex dynamical networks without state predictor.
\end{abstract}

\section{Introduction}

In recent years, the synchronization of complex dynamical networks has received more and more attention. The synchronous research can be applied in many fields, such as biology, smart city, computer, and the traffic [1-23].

It is well known that the complex network has a lot of nodes; however, in order to save an increasing number of energies, the pinning control is introduced to study the synchronization of complex dynamical networks. So far, the pinning control is a main tool by controlling a small number of nodes to steer the whole network. In [2], the pinning control of a continuous-time complex dynamical network with general coupling topologies was researched. The speed of synchronization is a significant issue, so, in [6], a state predictor was introduced. In [7], the adaptive synchronization of complex dynamical networks with state predictor was studied, therefore, this paper studies the problem using the pinning control.

This paper considers the adaptive synchronization of general complex dynamic networks via state predictor based on the fixed topology for nonlinear dynamical systems. With the limited information, state predictor can predict the future state of the nodes and its neighbors; therefore, general complex dynamic networks via state predictor can be faster to achieve synchronization.
This paper is organized as follows. Section 2 gives a model of the complex dynamical network. In addition, some preliminaries are introduced to prove the adaptive synchronization. Section 3 gives the main results and the theoretical analysis. The simulations of the theoretical results are given in Section 4. Finally, the conclusion is drawn in Section 5.

\section{Preliminaries and Problem Statement}

Consider a complex dynamical network described by

$$
\begin{aligned}
\dot{x}_{i}(t)= & f\left(x_{i}(t)\right)+\sum_{j=1}^{N} a_{i j} c_{i j}(t)\left[x_{j}(t)-x_{i}(t)\right] \\
& +\gamma \sum_{j \in \mathcal{N}_{i}} a_{i j} c_{i j}(t)\left(\dot{x}_{i}^{p}(t)-\dot{x}_{j}^{p}(t)\right)+u_{i}(t),
\end{aligned}
$$

where $x_{i}(t)=\left(x_{i 1}(t), x_{i 2}(t), \ldots, x_{i n}(t)\right)^{T} \in R^{n}(i=1,2$, $\ldots, N)$ is the state vector of the $i$ th node at time $t$, where $t$ is the continuous time; $f_{i}: R^{n} \rightarrow R^{n}$ is a continuous function; $N_{i}$ represents the neighbor node of $i ; a_{i j}$ typify the coupling weight between any two nodes, where $a_{i j} \geq 0$ and $a_{i i}=0 ; c_{i j}(t)$ stands for the coupling strengths between node $i$ and node $j$; 
define the matrix of the weighted coupling configuration of the system as

$$
U=\left[\begin{array}{cccc}
a_{11} c_{11} & a_{12} c_{12} & \cdots & a_{1 N} c_{1 N} \\
a_{21} c_{21} & a_{22} c_{22} & \cdots & a_{2 N} c_{2 N} \\
\vdots & \vdots & \ddots & \vdots \\
a_{N 1} c_{N 1} & a_{N 2} c_{N 2} & \cdots & a_{N N} c_{N N}
\end{array}\right] \in R^{N \times N}
$$

with $a_{i i} c_{i i}=-\sum_{j=1, j \neq i}^{N} a_{i j} c_{i j}$.

Introduce the state predictor as

$$
\dot{X}^{p}=-L X,
$$

where $\dot{X}^{p}=\left(\dot{x}_{1}^{p}, \dot{x}_{2}^{p}, \ldots, \dot{x}_{N}^{p}\right)^{T}, \gamma$ represents the impact factor of the state predictor.

Under the state predictor (3), network (1) can be written as

$$
\begin{aligned}
\dot{x}_{i}(t)= & f\left(x_{i}(t)\right)+\sum_{j=1}^{N} a_{i j} c_{i j}(t)\left[x_{j}(t)-x_{i}(t)\right] \\
& -\gamma\left[\sum_{j \in \mathcal{N}_{i}} \sum_{k \in \mathcal{N}_{i}} a_{i j} a_{i k} c_{i j}(t) c_{i k}(t)\left(x_{i}(t)-x_{k}(t)\right)\right. \\
& \left.\quad-\sum_{j \in \mathcal{N}_{i}} \sum_{p \in \mathcal{N}_{j}} a_{i j} a_{j p} c_{i j}(t) c_{j p}(t)\left(x_{j}(t)-x_{p}(t)\right)\right] \\
+ & u_{i}(t) .
\end{aligned}
$$

The control input is designed as

$$
u_{i}=-h_{i} c_{i}\left(x_{i}(t)-\bar{x}(t)\right),
$$

where $h_{i}$ is a binary number; if the $i$ th agent is controlled, $h_{i}=$ 1 ; otherwise $h_{i}=0 . c_{i}$ is the feedback gain of position.

Definition 1. Network (4) is said to achieve synchronization if

$$
\lim _{t \rightarrow \infty}\left\|x_{i}(t)-\bar{x}(t)\right\|=0, \quad i=1 \cdots N,
$$

where the homogeneous state satisfies

$$
\dot{\bar{x}}(t)=f(\bar{x}(t), t)=0 .
$$

The adaptive control at node $i$ is designed as

$$
\dot{c}_{i j}(t)=a_{i j} k_{i j}\left[x_{i}(t)-x_{j}(t)\right]^{T} P\left[x_{i}(t)-x_{j}(t)\right],
$$

where $c_{i j}(0) \geq 0$.

In the following, some necessary assumptions and lemmas are stated.

Assumption 2 (see [10]). The continuous function $f_{i}: R^{n} \times$ $[0,+\infty] \rightarrow R^{n}$ satisfies

$$
\begin{aligned}
& (x-y)^{T} P\{[f(x, t)-f(y, t)]-\Delta(x-y)\} \\
& \leq-\omega(x-y)^{T}(x-y),
\end{aligned}
$$

for $\forall x, y \in R^{n}$. And

$$
\begin{aligned}
& \Delta=\operatorname{diag}\left\{\delta_{1}, \ldots, \delta_{n}\right\}, \\
& P=\operatorname{diag}\left\{p_{1}, \ldots, p_{n}\right\}
\end{aligned}
$$

are positive constant matrices, for the constant $\omega>0$.

Lemma 3 (see [8]). For any vectors $x, y \in R^{n}$ and positivedefinite matrix $G \in R^{n \times n}$, the following matrix inequality holds:

$$
2 x^{T} y \leq x^{T} G x+y^{T} G^{-1} y .
$$

Lemma 4 (see [9]). Suppose that $a$ and $b$ are vectors; then for any positive-definite matrix $E$, the following inequality holds:

$$
-2 a^{T} b \leq \inf _{E>0}\left\{a^{T} E a+b^{T} E^{-1} b\right\} .
$$

Lemma 5 (see [10]). The following equation holds:

$$
\begin{aligned}
& \sum_{i=1}^{N}\left(x_{i}-\bar{x}\right)^{T} P \sum_{j=1, j \neq i}^{N} a_{i j} c_{i j}\left(x_{i}-x_{j}\right) \\
& =\frac{1}{2} \sum_{i=1}^{N} \sum_{j=1, j \neq i}^{N} a_{i j} c_{i j}\left(x_{i}-x_{j}\right)^{T} P\left(x_{i}-x_{j}\right) .
\end{aligned}
$$

Lemma 6 (see [18]). For a connected graph which is undirected, the Laplace matrix is positive semidefinite matrix, and the minimum nonzero eigenvalue is the algebraic connectivity of $L$, as follows:

$$
\lambda_{2}(L)=\min _{x \neq 0,1^{T} x=0} \frac{x^{T} L x}{\|x\|^{2}} .
$$

Lemma 7 (see [18]). For a system which is similar to $\dot{x}_{i}=$ $u_{i}(i=1,2, \ldots, n)$, the evolution rate associated with the minimum nonzero eigenvalue $\lambda_{2} . \lambda_{2}$ describes the lower bound of convergence rate. Generally, the bigger the $\lambda_{2}$ is, the faster the system converges.

\section{Main Results}

In the following, we will give the main result.

Theorem 8. Consider network (4) with the state predictor (3) and $N$ nodes steered by adaptive control (8), under Assumption 2 , and at least one node is selected to be controlled. Then, all nodes asymptotically synchronize with the given homogeneous stationary state:

$$
\lim _{t \rightarrow \infty}\left\|x_{i}(t)-\bar{x}(t)\right\|=0 .
$$

Proof. Let $\tilde{x}_{i}(t) \triangleq x_{i}(t)-\bar{x}(t)$. Construct the following Lyapunov function:

$$
V(t)=V_{1}(t)+V_{2}(t),
$$


where

$$
\begin{gathered}
V_{1}(t)=\frac{1}{2} \sum_{i=1}^{N} \widetilde{x}_{i}^{T}(t) P \widetilde{x}_{i}(t), \\
V_{2}(t)=\frac{1}{2} \sum_{i=1}^{N} \sum_{j \in \mathcal{N}_{i}} \frac{\left[c_{i j}(t)-m\right]^{2}}{2 k_{i j}} .
\end{gathered}
$$

Then

$$
\begin{aligned}
& \dot{V}_{1}(t)=\sum_{i=1}^{N} \tilde{x}_{i}^{T} P\left[f\left(x_{i}(t)\right)+\sum_{j=1}^{N} a_{i j} c_{i j}(t)\left(x_{j}-x_{i}\right)\right. \\
& +\gamma \sum_{j \in \mathcal{N}_{i}} a_{i j} c_{i j}(t)\left(\dot{x}_{i}^{p}(t)-\dot{x}_{j}^{p}(t)\right) \\
& \left.+u_{i}(t)-f(\bar{x}(t))\right] \\
& =\sum_{i=1}^{N} \tilde{x}_{i}^{T} P\left[f\left(x_{i}(t)\right)+\sum_{j=1}^{N} a_{i j} c_{i j}(t)\left(x_{j}-x_{i}\right)\right. \\
& -\gamma\left[\sum_{j \in \mathcal{N}_{i}} \sum_{k \in \mathcal{N}_{i}} a_{i j} a_{i k} c_{i j}(t) c_{i k}(t)\left(x_{i}-x_{k}\right)\right. \\
& -\sum_{j \in \mathcal{N}_{i}} \sum_{p \in \mathscr{N}_{j}} a_{i j} a_{j p} c_{i j}(t) c_{j p}(t) \\
& \left.\times\left(x_{j}-x_{p}\right)\right] \\
& \left.-h_{i} c_{i} \widetilde{x}_{i}(t)-f(\bar{x}(t))\right] \\
& =\sum_{i=1}^{N} \tilde{x}_{i}^{T} P\left[f\left(x_{i}(t)\right)-f(\bar{x}(t))\right] \\
& +\sum_{i=1}^{N} \widetilde{x}_{i}^{T} P \sum_{j=1}^{N} a_{i j} c_{i j}(t)\left(x_{j}-x_{i}\right) \\
& -\sum_{i=1}^{N} \tilde{x}_{i}^{T} P h_{i} c_{i} \tilde{x}_{i}^{T}-\gamma \sum_{i=1}^{N} \tilde{x}_{i}^{T} P \\
& \times \sum_{j \in \mathcal{N}_{i}} \sum_{k \in \mathcal{N}_{i}} a_{i j} a_{i k} c_{i j}(t) c_{i k}(t)\left(\tilde{x}_{i}-\tilde{x}_{k}\right) \\
& +\gamma \sum_{i=1}^{N} \tilde{x}_{i}^{T} P \sum_{j \in \mathcal{N}_{i}} \sum_{p \in \mathcal{N}_{j}} a_{i j} a_{i p} c_{i j}(t) c_{i p}(t)\left(\tilde{x}_{j}-\tilde{x}_{p}\right)
\end{aligned}
$$

$$
\begin{aligned}
\leq & -\omega \sum_{i=1}^{N} \tilde{x}_{i}^{T} \widetilde{x}_{i}+\sum_{i=1}^{N} \widetilde{x}_{i}^{T} P \Delta \widetilde{x}_{i} \\
& +\sum_{i=1}^{N} \widetilde{x}_{i}^{T} P \sum_{j=1}^{N} a_{i j} c_{i j}(t)\left(x_{j}-x_{i}\right) \\
& -\sum_{i=1}^{N} \widetilde{x}_{i}^{T} P h_{i} c_{i} \widetilde{x}_{i}^{T}-\gamma \sum_{i=1}^{N} \widetilde{x}_{i}^{T} P \\
& \times \sum_{j \in \mathcal{N}_{i}} \sum_{k \in \mathcal{N}_{i}} a_{i j} a_{i k} c_{i j}(t) c_{i k}(t) \tilde{x}_{i}
\end{aligned}
$$$$
+\gamma \sum_{i=1}^{N} \tilde{x}_{i}^{T} P \sum_{j \in \mathscr{N}_{i}} \sum_{k \in \mathcal{N}_{i}} a_{i j} a_{i k} c_{i j}(t) c_{i k}(t) \tilde{x}_{k}
$$$$
+\gamma \sum_{i=1}^{N} \widetilde{x}_{i}^{T} P \sum_{j \in \mathcal{N}_{i}} \sum_{p \in \mathcal{N}_{j}} a_{i j} a_{j p} c_{i j}(t) c_{j p}(t) \tilde{x}_{j}
$$$$
-\gamma \sum_{i=1}^{N} \widetilde{x}_{i}^{T} P \sum_{j \in \mathcal{N}_{i}} \sum_{p \in \mathcal{N}_{j}} a_{i j} a_{j p} c_{i j}(t) c_{j p}(t) \widetilde{x}_{p}
$$$$
\leq-\omega \sum_{i=1}^{N} \tilde{x}_{i}^{T} \widetilde{x}_{i}+\sum_{i=1}^{N} \widetilde{x}_{i}^{T} P \Delta \tilde{x}_{i}
$$$$
+\sum_{i=1}^{N} \widetilde{x}_{i}^{T}(t) P \sum_{j=1}^{N} a_{i j} c_{i j}(t)\left(x_{j}-x_{i}\right)
$$$$
-\sum_{i=1}^{N} \widetilde{x}_{i}^{T} P h_{i} c_{i} \tilde{x}_{i}-\gamma \sum_{i=1}^{N} \widetilde{x}_{i}^{T} P
$$$$
\times \sum_{j \in \mathscr{N}_{i}} \sum_{k \in \mathscr{N}_{i}} a_{i j} a_{i k} c_{i j}(t) c_{i k}(t) \tilde{x}_{i}
$$$$
+\frac{1}{2} \gamma \sum_{i=1}^{N} \sum_{j \in \mathcal{N}_{i}} \sum_{k \in \mathcal{N}_{i}} \tilde{x}_{i}^{T} P a_{i j} a_{i k} c_{i j}(t) c_{i k}(t) \tilde{x}_{i}
$$$$
+\frac{1}{2} \gamma \sum_{i=1}^{N} \sum_{j \in \mathcal{N}_{i}} \sum_{k \in \mathcal{N}_{i}} \tilde{x}_{k}^{T} P a_{i j} a_{i k} c_{i j}(t) c_{i k}(t) \tilde{x}_{k}
$$$$
+\frac{1}{2} \gamma \sum_{i=1}^{N} \sum_{j \in \mathscr{N}_{i}} \sum_{p \in \mathcal{N}_{j}} \tilde{x}_{i}^{T} P a_{i j} a_{j p} c_{i j}(t) c_{j p}(t) \tilde{x}_{i}
$$$$
+\frac{1}{2} \gamma \sum_{i=1}^{N} \sum_{j \in \mathcal{N}_{i}} \sum_{p \in \mathcal{N}_{j}} \tilde{x}_{j}^{T} P a_{i j} a_{j p} c_{i j}(t) c_{j p}(t) \tilde{x}_{j}
$$$$
+\frac{1}{2} \gamma \sum_{i=1}^{N} \sum_{j \in \mathcal{N}_{i}} \sum_{p \in \mathcal{N}_{j}} \tilde{x}_{i}^{T} P a_{i j} a_{j p} c_{i j}(t) c_{j p}(t) \tilde{x}_{i}
$$$$
+\frac{1}{2} \gamma \sum_{i=1}^{N} \sum_{j \in \mathcal{N}_{i}} \sum_{p \in \mathcal{N}_{j}} \tilde{x}_{p}^{T} P a_{i j} a_{j p} c_{i j}(t) c_{j p}(t) \tilde{x}_{p}
$$$$
=-\omega \sum_{i=1}^{N} \widetilde{x}_{i}^{T} \widetilde{x}_{i}+\sum_{i=1}^{N} \tilde{x}_{i}^{T} P \Delta \tilde{x}_{i}+\sum_{i=1}^{N} \tilde{x}_{i}^{T} P
$$ 


$$
\begin{aligned}
& \times \sum_{j=1}^{N} a_{i j} c_{i j}(t)\left(x_{j}-x_{i}\right) \\
& -\sum_{i=1}^{N} \tilde{x}_{i}^{T} P h_{i} c_{i} \tilde{x}_{i}-\frac{1}{2} \gamma \\
& \times \sum_{i=1}^{N} \sum_{j \in \mathcal{N}_{i}} \sum_{k \in \mathcal{N}_{i}} \tilde{x}_{i}^{T} P a_{i j} a_{i k} c_{i j}(t) c_{i k}(t) \tilde{x}_{i} \\
& +\frac{1}{2} \gamma \sum_{i=1}^{N} \sum_{j \in \mathcal{N}_{i}} \sum_{k \in \mathcal{N}_{i}} \tilde{x}_{k}^{T} P a_{i j} a_{i k} c_{i j}(t) c_{i k}(t) \tilde{x}_{k} \\
& +\gamma \sum_{i=1}^{N} \sum_{j \in \mathcal{N}_{i}} \sum_{p \in \mathcal{N}_{j}} \tilde{x}_{i}^{T} P a_{i j} a_{j p} c_{i j}(t) c_{j p}(t) \tilde{x}_{i} \\
& +\frac{1}{2} \gamma \sum_{i=1}^{N} \sum_{j \in \mathcal{N}_{i}} \sum_{p \in \mathcal{N}_{j}} \tilde{x}_{j}^{T} P a_{i j} a_{j p} c_{i j}(t) c_{j p}(t) \tilde{x}_{j} \\
& +\frac{1}{2} \gamma \sum_{i=1}^{N} \sum_{j \in \mathcal{N}_{i}} \sum_{p \in \mathcal{N}_{j}} \tilde{x}_{p}^{T} P a_{i j} a_{j p} c_{i j}(t) c_{j p}(t) \tilde{x}_{p} \\
& =-\omega \sum_{i=1}^{N} \tilde{x}_{i}^{T} \tilde{x}_{i}+\sum_{i=1}^{N} \tilde{x}_{i}^{T} P \Delta \tilde{x}_{i} \\
& +\sum_{i=1}^{N} \tilde{x}_{i}^{T} P \sum_{j=1}^{N} a_{i j} c_{i j}(t)\left(x_{j}-x_{i}\right) \\
& -\sum_{i=1}^{N} \tilde{x}_{i}^{T} P h_{i} c_{i} \tilde{x}_{i}+2 \gamma \sum_{i=1}^{N} \tilde{x}_{j}^{T} P L_{i}^{2} \tilde{x}_{j}
\end{aligned}
$$

where $L_{i}=\sum_{j=1, j \neq i}^{N} a_{i j} c_{i j}(t), i, j=1,2, \ldots, N$.

Consider the following:

$$
\begin{aligned}
& \dot{V}_{2}(t) \\
& =\frac{1}{2} \sum_{i=1}^{N} \sum_{j \in \mathcal{N}_{i}} \frac{\left(c_{i j}-m\right) \dot{c}_{i j}}{k_{i j}} \\
& =\frac{1}{2} \sum_{i=1}^{N} \sum_{j \in \mathcal{N}_{i}}\left(c_{i j}-m\right) a_{i j}\left[x_{i}-x_{j}\right]^{T} P\left[x_{i}-x_{j}\right] \\
& =\sum_{i=1}^{N} \tilde{x}_{i}^{T} P \sum_{j \in \mathcal{N}_{i}} a_{i j} c_{i j}\left(x_{i}-x_{j}\right) \\
& -m \sum_{i=1}^{N} \tilde{x}_{i}^{T} P \sum_{j \in \mathcal{N}_{i}} a_{i j}\left(x_{i}-x_{j}\right) . \\
& \dot{V}_{1}(t)+\dot{V}_{2}(t) \\
& \leq-\omega \sum_{i=1}^{N} \widetilde{x}_{i}^{T} \tilde{x}_{i}+\sum_{i=1}^{N} \widetilde{x}_{i}^{T} P \Delta \tilde{x}_{i}-\sum_{i=1}^{N} \tilde{x}_{i}^{T} P h_{i} c_{i} \tilde{x}_{i}
\end{aligned}
$$

$$
\begin{aligned}
& +2 \gamma \sum_{i=1}^{N} \tilde{x}_{j}^{T} P L_{i}^{2} \tilde{x}_{j}-m \sum_{i=1}^{N} \tilde{x}_{i}^{T} P \sum_{j \in \mathcal{N}_{i}} a_{i j}\left(\tilde{x}_{i}-\tilde{x}_{j}\right) \\
& =\tilde{x}^{T}\left[\left(I_{N} \otimes P \Delta+2 \gamma L^{2} \otimes P\right)\right. \\
& \quad-(H \otimes P+m A \otimes P)] \tilde{x}-\omega \sum_{i=1}^{N} \tilde{x}_{i}^{T} x_{i}^{T} .
\end{aligned}
$$

Since the positive constant $\mathrm{m}$ is sufficiently large, $\dot{V}(t)<$ 0 .

Therefore, $\lim _{t \rightarrow \infty}\left\|x_{i}(t)-\bar{x}(t)\right\|=0$.

Theorem 9. Network (4) with the state predictor (3) is faster to achieve synchronization than the network without the state predictor.

Proof. For the system with state predictor, the main difference is whether the system contains $\gamma \sum_{j \in \mathcal{N}_{i}} a_{i j} c_{i j}(t)\left(\dot{x}_{i}^{p}(t)-\dot{x}_{j}^{p}(t)\right)$. We consider the minimum nonzero eigenvalue of state predictor. The Laplace matrix is positive semidefinite matrix, so there is a nonsingular matrix $P$ that can make the Laplace matrix expressed as

$$
\gamma L^{2}=P^{-1}\left[\begin{array}{cccc}
\gamma \lambda_{n}^{2} & & & \\
& \ddots & & \\
& & \gamma \lambda_{2}^{2} & \\
& & & 0
\end{array}\right] P, \quad 0<\lambda_{2} \leq \cdots \leq \lambda_{n} .
$$

Obviously, under the same conditions, a system with state predictor has greater minimum nonzero eigenvalue. According to Lemma 7, network (4) with the state predictor (3) is faster to achieve synchronization than the network without the state predictor.

\section{Simulations}

In this section, a numerical simulation is given to illustrate the analytical results.

Consider a network with the undirected topology described as follows:

$$
A=\left[\begin{array}{cccc}
0 & 0.0964 & 0.0757 & 0.0570 \\
0.0964 & 0 & 0.1199 & 0.1396 \\
0.0757 & 0.1199 & 0 & 0.0581 \\
0.0570 & 0.1396 & 0.0581 & 0
\end{array}\right]
$$

where each node is a Lorenz system:

$$
f(x(t))=f\left(x^{1}, x^{2}, x^{3}\right)=\left\{\begin{array}{l}
\dot{x}^{1}=10\left(x^{2}-x^{1}\right) \\
\dot{x}^{2}=28 x^{1}-x^{1} x^{3}-x^{2} \\
\dot{x}^{3}=x^{1} x^{2}-\frac{8}{3} x^{3} .
\end{array}\right.
$$

Figure 1 describes the error states on the $x$-axis, $y$-axis, and $z$-axis, respectively. From Figure 1, we can see that all nodes can synchronize with the synchronous state by degrees. In particular, under the same conditions, the network with a state predictor can be synchronized faster. It is shown in Figure 2. 

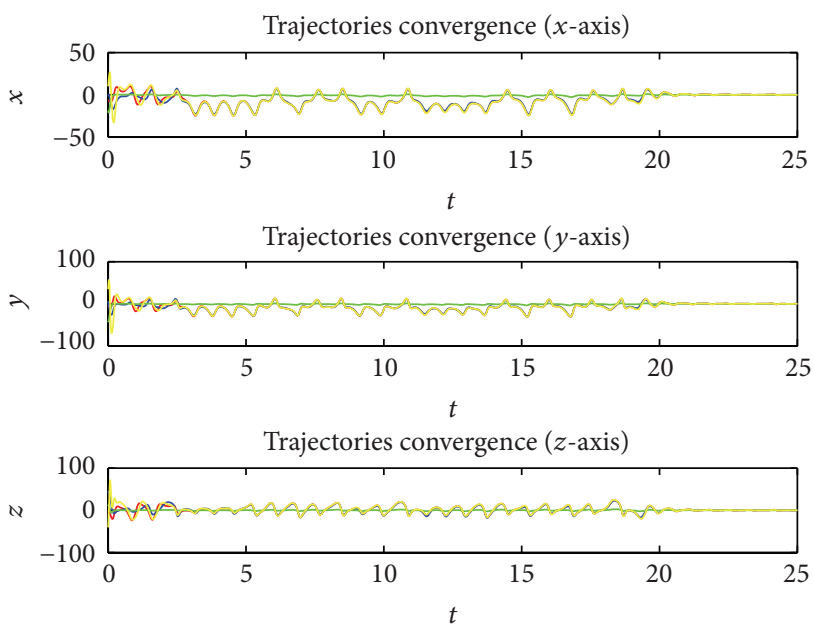

FIGURE 1: The dynamical network without a state predictor.
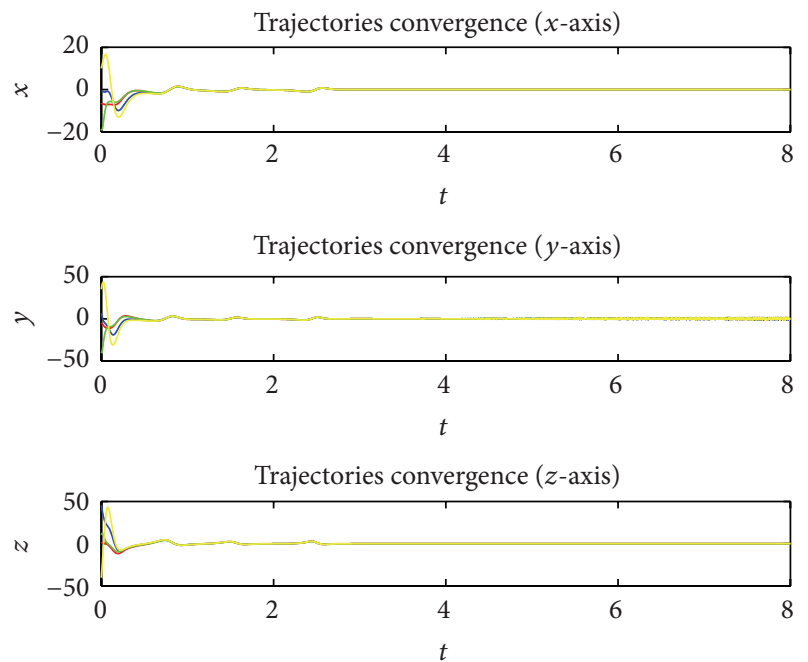

FIGURE 2: The dynamical network with a state predictor when $\gamma=$ 100.

\section{Conclusion}

In this paper, we have investigated the state predictor problem for synchronization of complex dynamical networks in fixed topology. By introducing local adaptive strategies for the coupling strengths, we have proved that the complex dynamical networks are asymptotically stable. It is obvious that the rate of convergence of the network with a state predictor is faster than the network without a state predictor.

\section{Conflict of Interests}

The authors declare that there is no conflict of interests regarding the publication of this paper.

\section{Acknowledgments}

This work was supported in part by the National Natural Science Foundation of China under Grant no. 61304049, Technology Development Plan Project of Beijing Education Commission (no. KM201310009011).

\section{References}

[1] W. Yu, G. Chen, J. Lü, and J. Kurths, "Synchronization via pinning control on general complex networks," SIAM Journal on Control and Optimization, vol. 51, no. 2, pp. 1395-1416, 2013.

[2] L. Y. Xiang, Z. X. Liu, Z. Q. Chen, F. Chen, and Z. Z. Yuan, "Pinning control of complex dynamical networks with general topology," Physica A: Statistical Mechanics and Its Applications, vol. 379, no. 1, pp. 298-306, 2007.

[3] X. F. Wang and G. Chen, "Pinning control of scale-free dynamical networks," Physica A, vol. 310, no. 3-4, pp. 521-531, 2002.

[4] P. de Lellis, M. di Bernardo, and F. Garofalo, "Synchronization of complex networks through local adaptive coupling," Chaos, vol. 18, no. 3, Article ID 037110, 8 pages, 2008.

[5] H. Su, Z. Rong, M. Z. Q. Chen, X. Wang, G. Chen, and H. Wang, "Decentralized adaptive pinning control for cluster synchronization of complex dynamical net works," IEEE Transactions on Systems, Man, and Cybernetics B: Cybernetics, vol. 43, no. 1, pp. 394-399, 2013.

[6] Y. Xi, W. Huang, and X. Li, "Consensus of multi-agent system with state predictor," Control and Decision, vol. 25, no. 5, pp. 769-772, 2010.

[7] Y. Shi, B. Liu, and X. Han, "Adaptive synchronization of complex dynamical networks with state predictor," Journal of Applied Mathematics, vol. 2013, Article ID 394137, 8 pages, 2013.

[8] H. Su, X. Wang, and Z. Lin, "Flocking of multi-agents with a virtual leader," IEEE Transactions on Automatic Control, vol. 54, no. 2, pp. 293-307, 2009.

[9] H. Su, N. Zhang, M. Z. Q. Chen, and X. Wang, "Adaptive flocking with a virtual leader of multiple agents governed by locally Lipschitz nonlinearity," Nonlinear Analysis: Real World Applications, vol. 14, no. 1, pp. 798-806, 2013.

[10] H. Su, G. Chen, X. Wang, and Z. Lin, "Adaptive secondorder consensus of networked mobile agents with nonlinear dynamics," Automatica, vol. 47, no. 2, pp. 368-375, 2011.

[11] B. Liu, W. Hu, J. Zhang, and H. Su, "Controllability of discretetime multi-agent systems with multiple leaders on fixed networks," Communications in Theoretical Physics, vol. 58, no. 6, pp. 856-862, 2012.

[12] H. Su, M. Z. Q. Chen, J. Lam, and Z. Lin, "Semi-global leaderfollowing consensus of linear multi-agent systems with input saturation via low gain feedback," IEEE Transactions on Circuits and Systems. I. Regular Papers, vol. 60, no. 7, pp. 1881-1889, 2013.

[13] B. Liu, X. Wang, and J. Zhang, "Adaptive pinning synchronization of the complex dynamical network," in Proceedings of the International Conference on Automatic Control and Artificial Intelligence, vol. 6, pp. 4173-4176, 2012.

[14] W. Yu, P. DeLellis, G. Chen, M. di Bernardo, and J. Kurths, "Distributed adaptive control of synchronization in complex networks," IEEE Transactions on Automatic Control, vol. 57, no. 8, pp. 2153-2158, 2012.

[15] W. Yu, G. Chen, and J. Lü, "On pinning synchronization of complex dynamical networks," Automatica, vol. 45, no. 2, pp. 429-435, 2009. 
[16] Z. Hou, L. Cheng, and M. Tan, "Decentralized robust adaptive control for the multiagent system consensus problem using neural networks," IEEE Transactions on Systems, Man, and Cybernetics B: Cybernetics, vol. 39, no. 3, pp. 636-647, 2009.

[17] P. de Lellis, M. di Bernardo, and F. Garofalo, "Synchronization of complex networks through local adaptive coupling," Chaos, vol. 18, no. 3, Article ID 037110, 2008.

[18] Y. Xi, W. Huang, and X. Li, "Consensus of multi-agent system with state predictor," Control and Decision, vol. 25, no. 5, pp. 669-772, 2010.

[19] B. Tadić, S. Thurner, and G. J. Rodgers, "Traffic on complex networks: towards understanding global statistical properties from microscopic density fluctuations," Physical Review E, vol. 69, no. 3, Article ID 036102, 2004.

[20] G. Xi, W. Huang, and X. Li, "Consensus of multi-agent system with state predictor," Control and Decision, vol. 25, no. 5, pp. 669-772, 2010.

[21] L. Zhao, Y. Lai, K. Park, and N. Ye, "Onset of traffic congestion in complex networks," Physical Review E-Statistical, Nonlinear, and Soft Matter Physics, vol. 71, no. 2, Article ID 026125, 2005.

[22] Z. Gao, D. Kong, and C. Gao, "Modeling and control of complex dynamic systems: applied mathematical aspects," Journal of Applied Mathematics, vol. 2012, Article ID 869792, 5 pages, 2012.

[23] Z. Gao, D. Kong, C. Gao, and M. Chen, "Modeling and control of complex dynamic systems," Journal of Applied Mathematics, vol. 2013, Article ID 151372, 3 pages, 2013. 


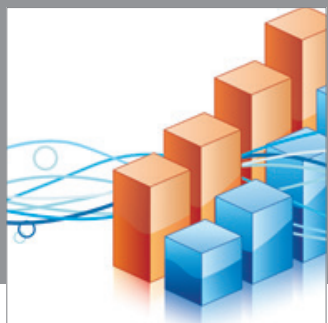

Advances in

Operations Research

mansans

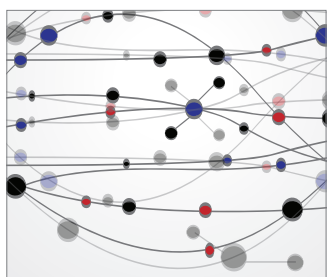

The Scientific World Journal
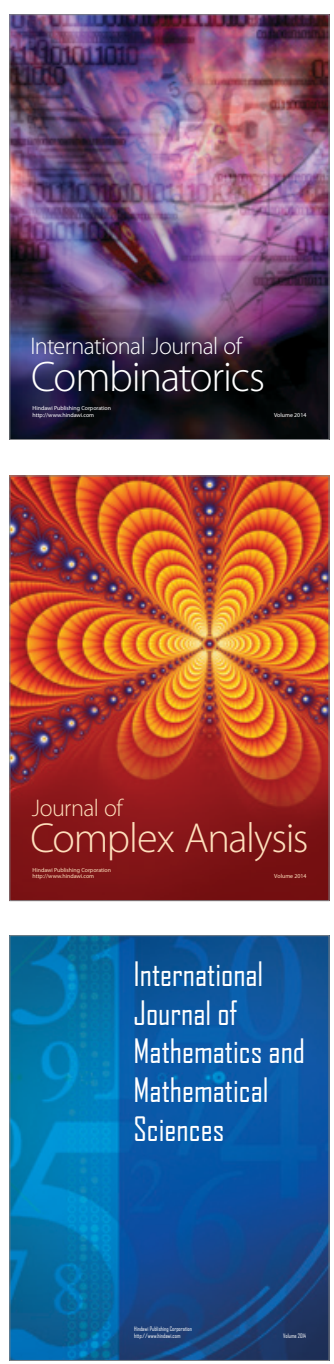
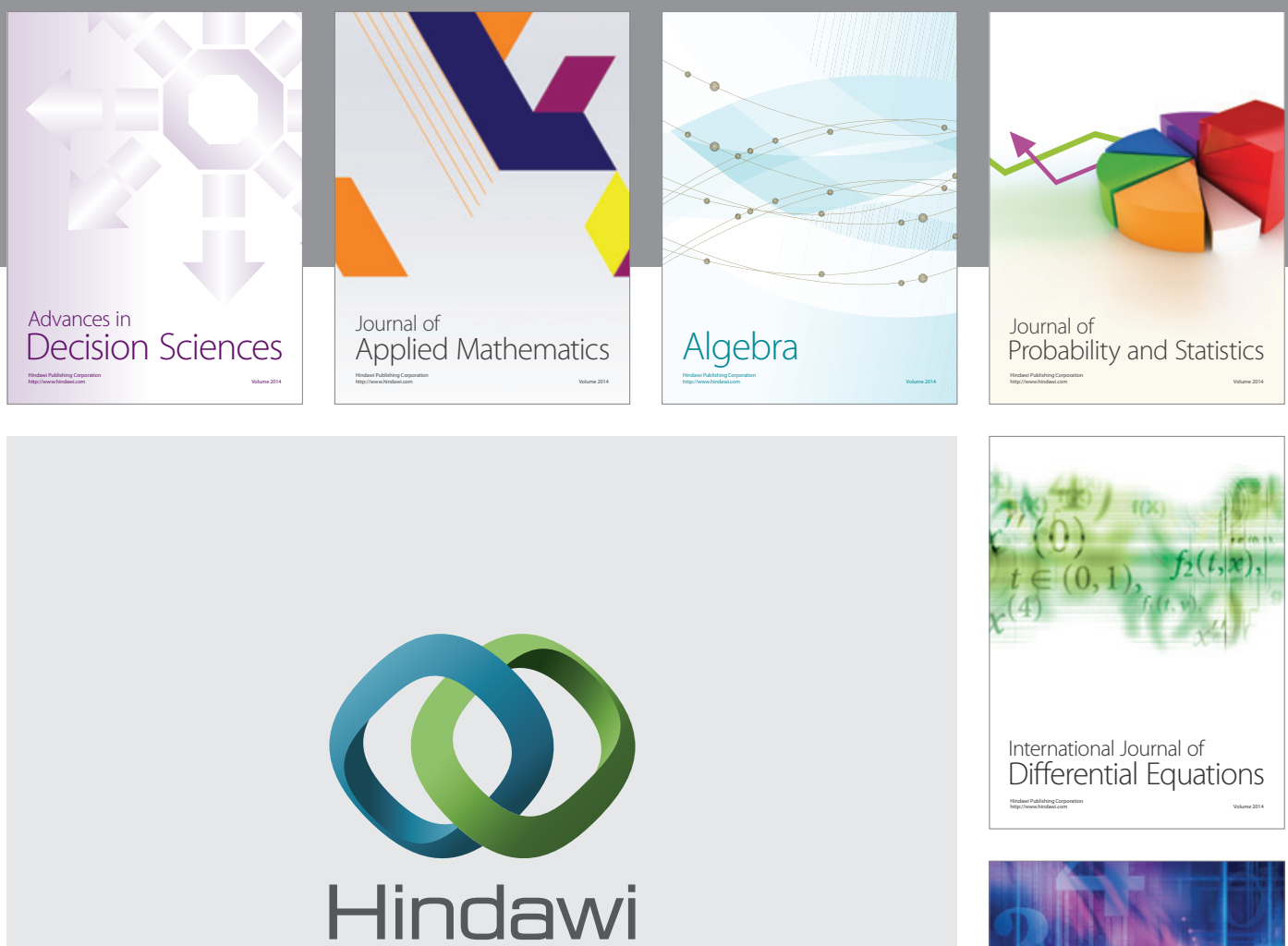

Submit your manuscripts at http://www.hindawi.com
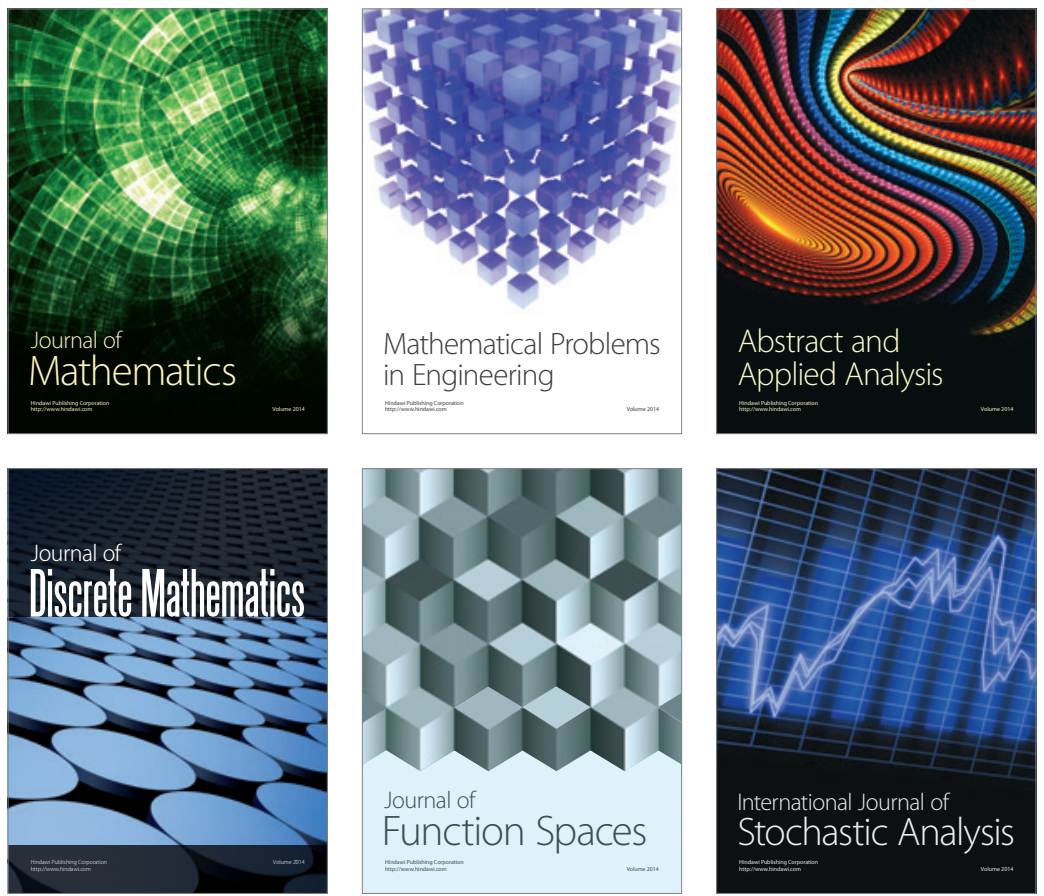

Journal of

Function Spaces

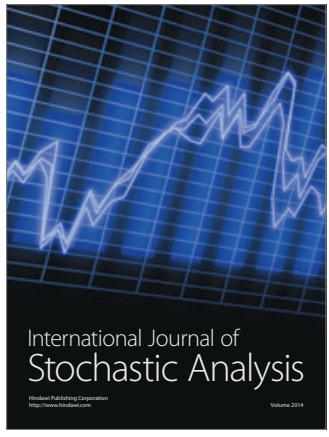

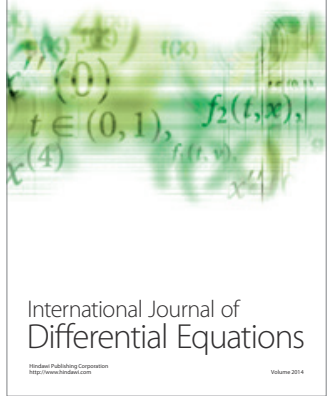
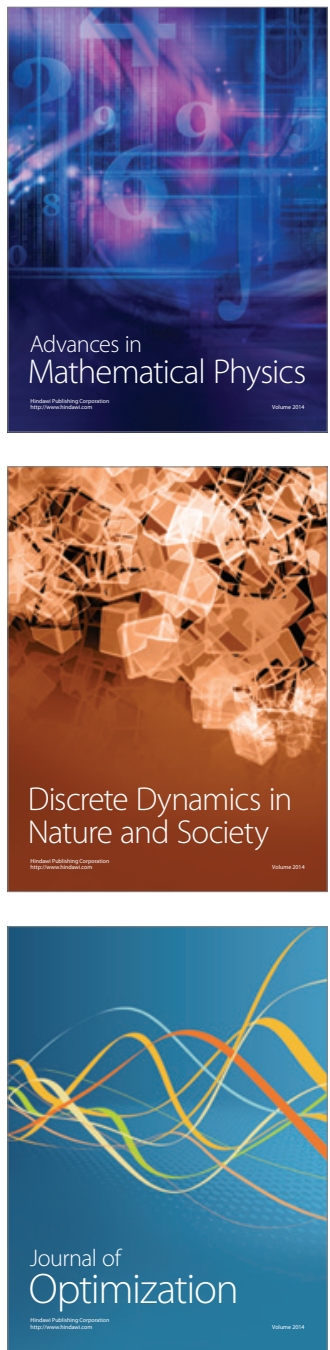